\title{
Multiple Frequency Ultrasounds Assisted Oil Sands Separation Technology
}

\author{
Kaili $\operatorname{LIAO}^{(1)}$, Jijiang GE ${ }^{(1)}$, Guicai ZHANG ${ }^{(1)}$, Lipei FU ${ }^{(2)}$ \\ (1) College of Petroleum Engineering, China University of Petroleum \\ Qingdao 266580, China; e-mail: lkl123@163.com, jijiangge@163.com, 13706368080@163.vip.163.com \\ (2) School of Petroleum Engineering, Changzhou University \\ Changzhou 213016, China \\ (received October 13, 2016; accepted July 26, 2017)
}

\begin{abstract}
Ultrasound has a wide range of applications in oil sands separation industry due to its green and nopolluting characteristics. The combined ultrasounds technology has been widely used in many industries by virtue of the synergistic effect of cavitation effect; meanwhile, dual-frequency ultrasounds have been reported being used in lotion oil sands technology. Based on this idea, this study focuses on the application of multiple frequency ultrasounds in oil sands separation, and a comparative study has been conducted between the combined ultrasounds systems with the difference in the number of the ultrasound. The results show that the oil production rate of the samples treated by the lotion of sodium dodecyl benzene sulfonate (SDBS) and assisted by multiple frequency ultrasounds (96\%) is significantly higher than that of the single frequency ultrasound $(76 \%)$; the bigger the number of the ultrasound participating in the combination is, the higher the oil production rate of the oil sands is; the lower the frequency of the ultrasounds employed in the combined system is, the higher the oil production rate is. The optimum treating conditions for tri-frequency ultrasounds assisted technology are as follow: the treating time is $10-15 \mathrm{~min}$, the treating temperature is $20-30^{\circ} \mathrm{C}$, the concentration of surfactant in the lotion is $1.5 \mathrm{~g} / \mathrm{l}$, and the mass ratio of the lotion to oil sands is 1.8. In short, the use of multiple frequency ultrasounds can improve the oil production rate of oil sands, reduce the energy consumption during the separation process, and reduce the environmental contamination; therefore, multiple frequency ultrasounds assisted oil sands separation technology is a promising technology for oil sands resources exploitation with high efficiency.
\end{abstract}

Keywords: multiple frequency ultrasounds; cavitation effect; oil sands separation; synergistic effect.

\section{Introduction}

As an unconventional energy resource, oil sands, or to say asphalt/bitumen sands, are mixture of asphalt/bitumen, sand/clay, and water, commonly with the proportion of $3-20 \%, 80-87 \%$, and $3-6 \%$ respectively (Kramers, Mossop, 1987). According to statistics, the oil sands resource reserves are about 400 Gt globally when it is converted to heavy oil, which is much larger than the reserves of the natural oil (200 Gt) (Yu et al., 2013; Kramers, Mossop, 1987). Therefore, oil sands resource is undeniable alternative energy sources. The oil sands can be divided into three types according to its structure: water wetting, oil wetting and medium wetting type (YAO, 2012). According to the Canadian standard, when the content of asphalt is more than $10 \%$, it can be classified as rich oil sands, and if the content of asphalt is less than $6 \%$, it can be classified as poor oil sands (CZARNECKI et al., 2005;
Yun et al., 2004). The oil sands resources in China are relatively rich, mainly distributed in provinces of Xinjiang, Qinghai, Tibet, Sichuan, and Inner Mongolia. The oil sands resources account for about $1 / 3$ of China's recoverable oil and gas reserves (JIA et al., 2012; CAO et al., 2012).

The chemical composition of the oil component in oil sands is extremely complex, and mainly consists of polycyclic aromatic hydrocarbon and macromolecular hydrocarbons. Asphalt oil has a high viscosity, relatively large molecular, and a high content of colloid and asphaltene (ZhANG et al., 2014; PATHAKA et al., 2011). The mobility and mutual solubility are poor, and are easy deposition, adsorption in contact with the interface. Therefore, separating the asphalt from oil sands is very difficult, and different separation methods should be used (Alturki et al., 2010; Gates, 2010; Rudyk, Spirov, 2014; Mutyala et al., 2010). The most widely used technique is water-based lotions 
separation method, which means that the surfactant or the alkali solution is used and mixed with the oil sands, and oil sands separation is done after mechanical stirring and centrifugal treating (ZHANG et al., 2008; Woods et al., 2012). Clark, as one of the first researchers, studied using alkaline to separate oil sands (Clark, Pasternack, 1932). Sanford pointed out that $\mathrm{NaOH}$ could neutralize the organic acid in the oil sands, during which the surfactant was produced, and increase the yield of the asphalt (SANFORD, SEvER, 1979). Schram studied oil sands separation with the surfactant solution, and pointed out the mechanism (YU et al., 2013). The surfactant can reduce the surface tension of oil and water, making the asphalt film on the sand be broken and shrinking along the surface of the sand to form asphalt droplets. The separated asphalt droplets can combine with the bubbles, float to the surface of the water, and form asphalt foam. Finally, the asphalt can be obtained after centrifugal separation.

In recent years, ultrasound, with the advantages of high efficiency and low energy consumption, has been widely used in oil sands industry. Through the study of Abramov, Feng, Zhao, and Zou, the mechanism of ultrasound assisted oil sands separation technology is summarized as follows (ABRAMOV, MYASNIKOV, 2009; FEnG, 2013; ZHAO et al., 2011; ZoU et al., 2016). Ultrasonic cavitation is the main mechanism of ultrasound assisted oil sands separation technology. The cavitation effect produces four additional effects in the process: turbulence effect, perturbation effect, energyaggregating effect, and interface effect. The interface effect is a mechanical effect generated by the acoustic flow and the micro jet generated at the interface between the oil sands and the water, which can accelerate the peeling of the oil sands surface; the energyaggregating effect and perturbation effect can reduce the adhesive stress between the asphalt and the sands; the turbulence effect produces a lot of vortices during the separation process, accelerating the emulsification of the tripped asphalt droplets. On the one hand, these four effects can reduce the thickness of asphalt and accelerate energy transfer between the liquid-solid medium during the separation process; on the other hand the ultrasonic waves can make the system of intense turbulence, which enhances the stirring action and the collapse of the asphalt. Therefore, the asphalt will separate from the surface of the oil sands (CAO, 2011).

Multi-frequency ultrasounds, i.e. when more than one ultrasound act on the sample at the same time, can produce a significant increase in cavitation activity compared with single frequency irradiation, and the cavitation production is much larger than the algebraic sum of that under individual irradiation. Due to the synergistic effect of the dual-frequency ultrasounds, the dual-frequency ultrasounds have been widely used in other industries such as the degradation of ammonia nitrogen in coked waste water and the disintegration of sludge aggregates (TAN, 2011; ZHANG, WU, 2010). Fu pointed out that the oil production rate of single frequency (83\%) is greatly improved to $95 \%$ of the dual-frequency ultrasounds. Beyond that, the new technology also improves the treating conditions of oil sands separation: a shorting of time of $66 \%$, a reduction of dosage of agent $60 \%$, and a fall of temperature of $40^{\circ} \mathrm{C}$ (FU et al., 2017). In this study, tri-frequency ultrasounds were applied to oil sands separation, and a comparative study was conducted between the combined ultrasounds systems with the difference in the number of the ultrasound.

\section{Experimental section}

\subsection{Materials and instruments}

The materials used in the experiment consisted of oil sands samples and four kinds of surfactant. The oil sands, with the component of $9.8 \%$ asphalt, $5.1 \%$ water, and $85.1 \%$ solid, were provided by oilfield. The surfactant, including sodium dodecyl benzene sulfonate (SDBS), sodium oleate (NaOL), and sodium Laurate (NaLA), were purchased from Sinopharm, China. The shengli petroleum sulfonate (SLPS) was obtained from oilfield. The mechanical agitating equipment was an overhead blender, with the stirring rate of $300 \mathrm{r} \mathrm{min}^{-1}$. The multiple frequency acoustic wave generator with the frequency of $28 \mathrm{kHz}, 68 \mathrm{kHz}$, and $80 \mathrm{kHz}$ was built by our research group.

\subsection{Experimental method}

The oil sands were put into the surfactant solution at a certain mass ratio. For the treating process without ultrasound, the mixture was treated by mechanical agitating equipment. For the treating process under the effect of ultrasound, the mixture was poured into the acoustic generator. The separation procedure was conducted according to the standard method for oil sands separation treated by water-based lotion as follows. The mixture which was treated was put into the flotation container equipped with an air inlet at the bottom of the container. Therefore, when the bubbles rose up from the bottom of the container, the microdroplets of asphalt separated from the solid particles met with the bubbles and formed asphalt foam. The asphalt foam came up to the surface of the solution and could be collected by decantation method. The procedure was repeated until no more asphalt foam floated up. The asphalt and water were separated when the defoamer was added. Finally, the micro fines were completely separated from the asphalt by the method of extraction and centrifugation. The oil production rate was defined as the ratio between the amount of the 
produced oil from unit mass of oil sands and the oil content of the unit mass of oil sands.

The multiple frequency ultrasounds assisted oil sands separation technology means using a group of ultrasounds with different frequencies to treat the oil sands sample. In the experiment, dual-frequency ultrasounds with the combined mode of $28 \mathrm{kHz}$ and $68 \mathrm{kHz}, 28 \mathrm{kHz}$ and $80 \mathrm{kHz}$, or $68 \mathrm{kHz}$ and $80 \mathrm{kHz}$, and tri-frequency ultrasounds with the combined mode of $28 \mathrm{kHz}$ and $68 \mathrm{kHz}$ and $82 \mathrm{kHz}$ were studied. And they are abbreviated as combined ultrasounds (28/68), combined ultrasounds $(28 / 80)$, combined ultrasounds $(68 / 80)$, and combined ultrasounds (28/68/80). In addition, ultrasound of $28 \mathrm{kHz}$ means using the ultrasound with the frequency of $28 \mathrm{kHz}$ to treat the oil sands sample.

\section{Results and discussion}

\subsection{Determination of the formula of the lotion}

During the ultrasounds assisted oil sands separation process, the formula of the lotion directly affects the oil production rate. Therefore, experiments were conducted to determine the concentration of the surfactant solution, as well as its dosage, or the mass ratio of the lotion to the oil sands sample. Four types of surfactant were used. During the separation process, the temperature was $20^{\circ} \mathrm{C}$, and the treating time was 45 min.

The relationship of the concentration of the surfactant solution and the oil production rate is shown in Fig. 1. What needs to be pointed out is that the mass ratio of the surfactant solution to oil sands sample was $2: 1$ during the concentration optimization experiments. It can be seen from Fig. 1 that when the concentration of the surfactant solution was lower than $2 \mathrm{~g} / \mathrm{l}$, the oil production rate was very low, which is

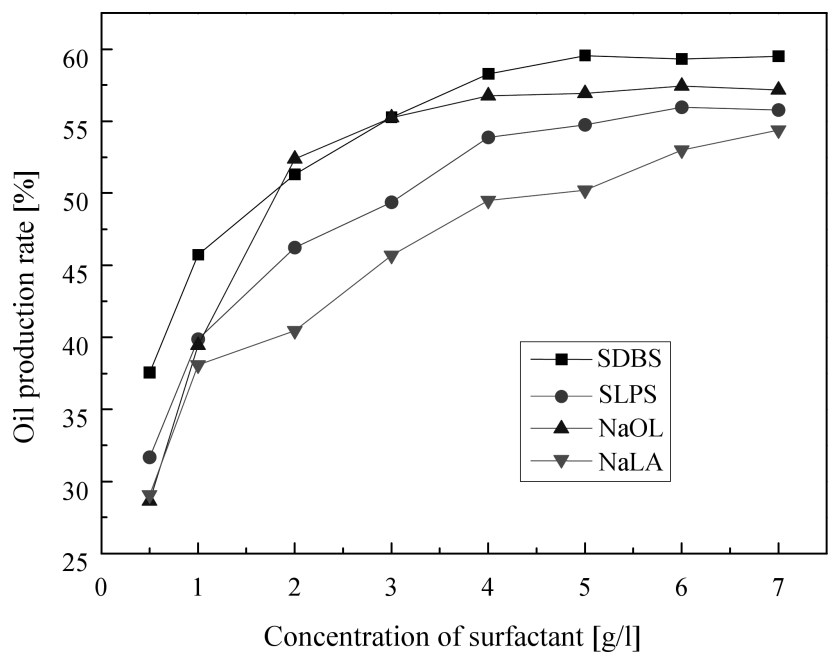

Fig. 1. Relationship of the oil production rate and the concentration of surfactant. also applied to all the four types of surfactant. From the curves, we can see that the oil production rate tends to be stable with the increase in the concentration of the surfactant solution. Meanwhile, when the concentration is $5 \mathrm{~g} / \mathrm{l}$, the oil production rates of the SDBS, SLPS, NaOL, and NaLA is $59.56 \%, 54.74 \%$, $56.93 \%$, and $50.22 \%$ respectively, indicating that the surfactant of SDBS and NaOL exhibits stronger ability in oil sands separation than the other two types of surfactant. Therefore, the mentioned two types of surfactant with the concentration of $5 \mathrm{~g} / \mathrm{l}$ were selected for the further research.

The surfactant solution of SDBS and NAOL was used to study the relationship of the oil production rate with the mass ratio of the lotion to oil sands, and the results are shown in Fig. 2. It can be seen that the oil production rate increases with the increase in mass ratio of lotion to oil sands. When comes to SDBS, the optimum mass ratio which exhibits the largest oil production rate is 2.4 , and the oil production rate is $64.47 \%$. From the curve of $\mathrm{NaOL}$, it can be obtained that the oil production rate tends to steady when the mass ratio is 2.0, exhibiting that the NaOL has stronger asphalt removing ability compared with the same amount of SDBS solution. However, the maximum oil production rate is lower than that of the SDBS solution. Therefore, the lotion for oil sands separation is determined as the solution of SDBS, with the concentration of $5 \mathrm{~g} / \mathrm{l}$, and the mass ratio of the lotion to oil sands is 2.4 .

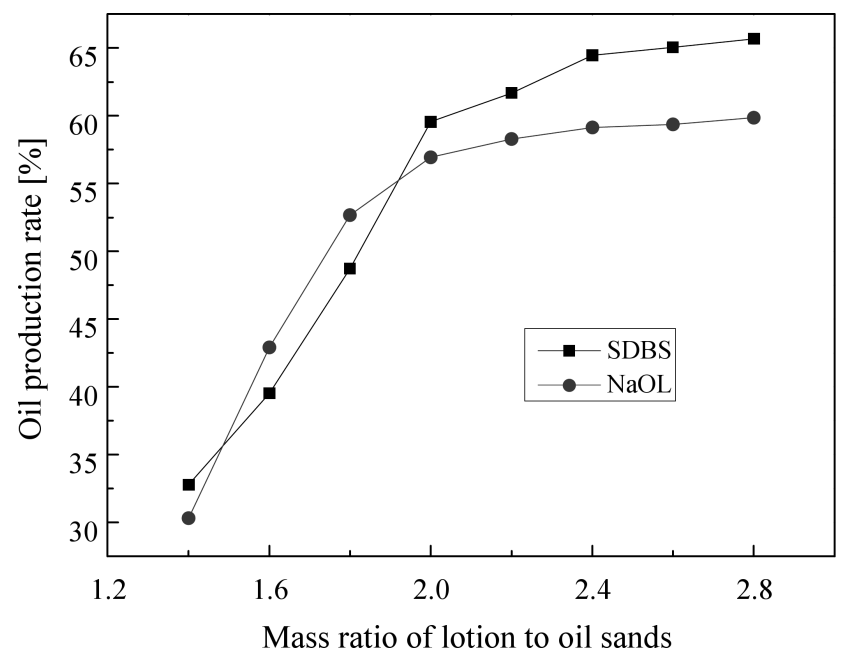

Fig. 2. Relationship of the oil production rate and the mass ratio of the lotion to oil sands.

\subsection{Effect of combined ultrasounds on oil production rate}

\subsubsection{Effect of ultrasonic frequency on oil production rate}

The ultrasounds with different frequencies were employed for oil sands separation in order to study the ultrasonic frequency on oil production rate. The exper- 
iments were conducted under the condition that: the determined surfactant solution was used, the temperature was $20^{\circ} \mathrm{C}$, and the treating time was $45 \mathrm{~min}$. The tests which were treated under the mechanical stirring were carried out as compare experiments. The results are shown in Fig. 3.

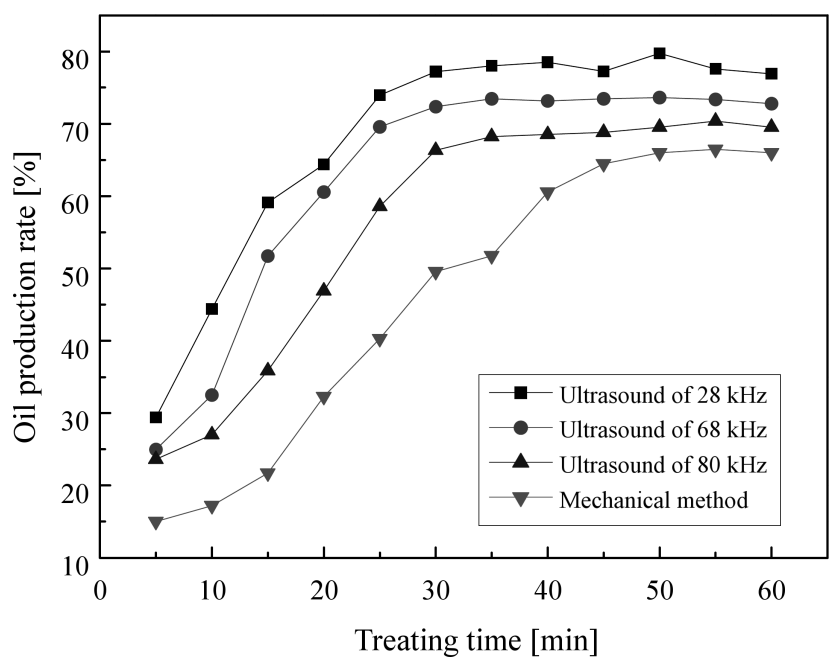

Fig. 3. Effect of the ultrasonic frequency on the oil production rate.

Three conclusions can be drawn from Fig. 3. The first one is that the oil production rate increases with the increase in treating time, and finally the curves tend to steady. The curves show that the maximum oil production rates of the samples treated by ultrasound with the frequency of $28 \mathrm{kHz}, 68 \mathrm{kHz}$, and $80 \mathrm{kHz}$, and without ultrasound are $77.21 \%, 72.36 \%, 66.33 \%$, and $64.47 \%$, respectively. Therefore, the second conclusion is that the oil sands samples treated under the effect of ultrasound have higher oil production rate than the samples treated without ultrasound. The third one is that the higher of the ultrasonic frequency is, the higher the oil production rate becomes.

The reasons for the second conclusion are as follows. The separation stress of the mechanical stirring mainly depends on the tangential velocity, and the turbulence effect is relatively weak compared to the effect generated by ultrasound. In addition, the oil sands are still mainly in the form of block-shape during the mechanical action, and the molecules of SDBS cannot contact with the internal sample effectively, so that the oil production rate is lower than that of the samples treated with ultrasound. When comes to ultrasound, ultrasonic cavitation is the main mechanism of ultrasound assisted oil sands separation technology. The cavitation effect will produce four additional effects in the process: turbulence effect, perturbation effect, energy-aggregating effect, and interface effect. On the one hand, cavitation effect can reduce the thickness of asphalt and accelerate energy transfer between the liquid-solid medium during the separation process; on the other hand, because the ultrasonic waves can make the system of intense turbulence, which enhances the stirring action and the collapse of the asphalt. Therefore, the asphalt will separate from the surface of the oil sands efficiently. The reason supporting the third conclusion is that with the increase of the acoustic frequency, the cavitation effect of the ultrasound weakens, as well as the turbulence effect generated by the ultrasound, resulting in lower oil production rate.

\subsubsection{Effect of combination ways on oil production rate}

Research shows that the dual-frequency ultrasounds exhibit better ability in oil sands separation. Tri-frequency ultrasounds were introduced to this technology, and a comparative study was conducted between the combined ultrasounds systems with the difference in the number of the ultrasound. The experimental conditions were the same as the tests conducted to study the effect of ultrasonic frequency on oil production rate. The results are shown in Fig. 4.

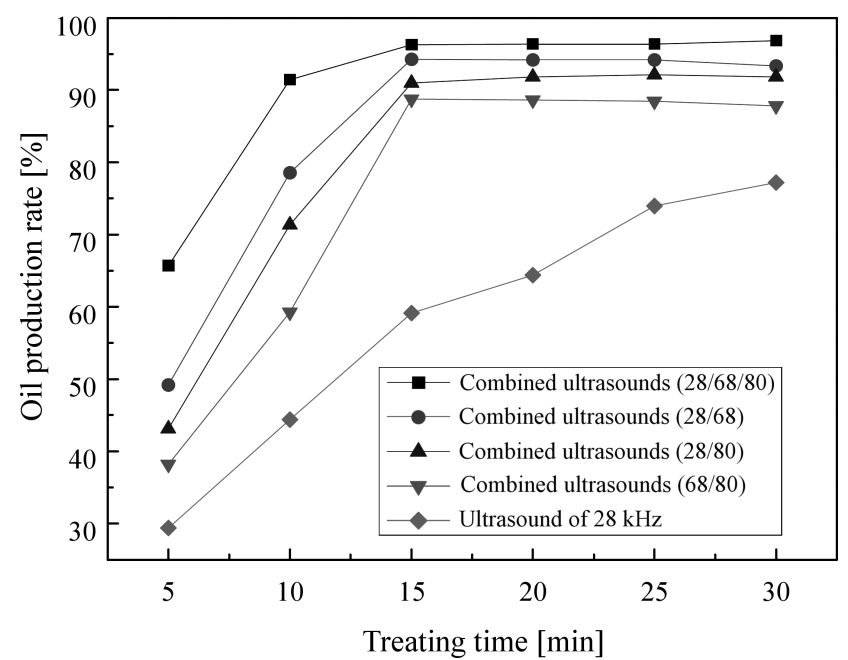

Fig. 4. Effect of combination ways of the ultrasounds on oil production rate.

It can be drawn from the curves that the oil production rates of the samples treated by multiple ultrasounds are higher than that of the samples treated by single ultrasound. The reason lies in the fact that when the samples are treated by multiple frequency ultrasounds, more than one ultrasound influences the samples. The cavitation effects of the ultrasounds produce synergistic effects, resulting in stronger cavitation effect than single frequency ultrasound. The mechanism behind the synergistic effects of the cavitation effects is that the cavitation bubbles produced by different ultrasounds can be used not only by their own ultrasound but also by other ultrasounds. In addition, because the collapse time of the cavitation bubbles generated by multiple frequency ultrasounds is shortened, more times of the collapse occurs, resulting in higher oil production rate. 
In addition, the oil sands samples treated by tri-frequency ultrasounds exhibit stronger ability in oil sands separation than dual-frequency ultrasounds. This result can be attributed to the reasons shown in the last paragraph. Furthermore, it can be seen from the curves of combined ultrasounds (28/68) and combined ultrasounds $(68 / 80)$ that the oil production rates of the former are higher than that of the latter. This phenomenon can be explained by the mechanism that the lower the ultrasonic frequency is, the stronger the cavitation effect becomes, and the higher of the oil production rate will be.

\subsection{Evaluation of the reaction conditions under the effect of combined ultrasounds}

\subsubsection{Effect of treating time on oil production rate}

From the analysis mentioned above, the conclusion that the introduction of multiple frequency ultrasounds into oil sands separation by lotion increases the oil production rate of oil sands, can be drawn. However, in industrial applications, other issues should be taken into consideration, such as the treating time, the lotion consumption, and the treating temperature. The relationships of the oil production rate to the treating time of multiple frequency ultrasounds are shown in Fig. 4. With the increase in the treating time of the ultrasounds, the oil production rate increases gradually, and finally becomes stable; for the single frequency ultrasound, it needs about $30 \mathrm{~min}$ to achieve the maximum oil production rate, while for multiple frequency ultrasounds, it takes about $15 \mathrm{~min}$ to achieve the maximum oil production rate, indicating that the multiple frequency ultrasounds speed up the process of oil sands separation; it takes 15 min for tri-frequency ultrasounds to achieve the maximum value, however, the oil production rate can reach $95 \%$ of the maximum value if the oil sands were treated by $10 \mathrm{~min}$, which is different from dual-frequency ultrasounds, indicating that the number of the ultrasounds in the multiple frequency ultrasounds system has an effect on the speed of the oil sands separation.

The reasons for the mentioned results are as follows. The prolongation of the treating time of the ultrasounds results in the accumulation of the cavitation effect of the ultrasounds, under the action of which the mass of the oil sands being separated increases. Therefore, the oil production rate increases. In addition, due to the synergistic effect of the cavitation effect, the cavitation effects generated in the acoustic field of the multiple frequency ultrasounds are stronger than in the acoustic field of single frequency ultrasound. Thus, the oil production rate of the former is higher than that of the latter under the process of the same time treatment. This mechanism is also applicable to multiple frequency ultrasounds, which can be employed to explain that the oil production rates of the oil sands treated by tri-frequency ultrasounds are higher than the dual-frequency ultrasounds.

\subsubsection{Effect of the formula and dosage of lotion on oil production rate}

In order to determine the effect of the lotion on oil sands separation, experiments of the influence of the dosage and the concentration of the lotion on oil production rate have been conducted. The tests were conducted under the conditions that the treating time was $45 \mathrm{~min}$, and the temperature was $20^{\circ} \mathrm{C}$. The relationships of the oil production rates with the concentration of surfactant under different treating conditions are shown in Fig. 5. It can be seen from Fig. 5 that the oil production rates increase with the increase in the concentration of surfactant. The mechanism for oil sands separation under the effect of surfactant is that the surfactant molecule can reduce the interfacial tension and make the asphalt to be peeled from the sands easily. The ability to reduce the interfacial tension is weak when the concentration of lotion is low, so the oil production rate is low. When the concentration of surfactant reaches a certain degree, the ability in interfacial tension improvement tends to be stable. Therefore, the oil production increases with the increase in lotion concentration, and eventually stabilized. The optimum concentration of surfactant to achieve the maximum oil production rate for samples treated by trifrequency ultrasounds is $1.5 \mathrm{~g} / \mathrm{l}$, while $2 \mathrm{~g} / \mathrm{l}$ and $4 \mathrm{~g} / \mathrm{l}$ for the samples treated by dual-frequency ultrasounds and single frequency ultrasound, respectively. The reason for these phenomena is that the synergistic cavitation effect of multiple frequency ultrasounds can produce strong mechanical perturbation effect inside the lotion, which accelerates the separating stress of asphalt from sands, enhances the transmission efficiency in the liquid-solid system of the acoustic energy, and increases the oil production rate.

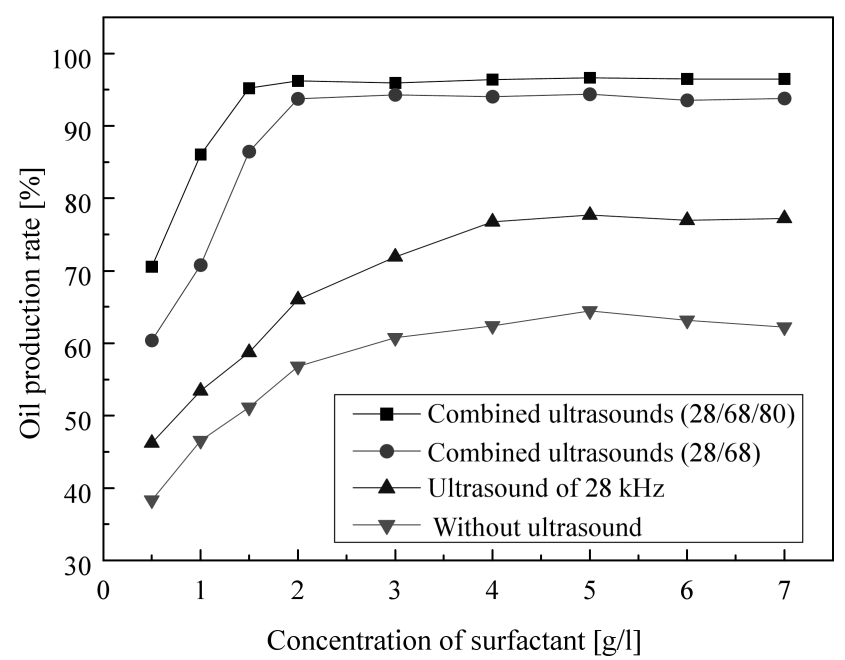

Fig. 5. Effect of concentration of surfactant on oil production rate. 
The relationship of the oil production rates to the mass ratio of the lotion to oil sands under different treating conditions is shown in Fig. 6. It can be seen from Fig. 6 that the optimum mass ratio for combined ultrasounds $(28 / 68 / 80)$, combined ultrasounds (28/68), ultrasound of $28 \mathrm{kHz}$, and without ultrasound are 1.8, 2.0, 2.4, and 2.4 respectively. The premise for oil sands separation treated by lotion is that the surfactant molecule in the lotion is in full contact with each oil sand particles. When the amount of lotion is less, the solid and liquid cannot form an effective contact, and the incomplete reaction makes it difficult to strip the oil droplets off the sand surface. However, the cavitation effect generated by the multiple frequency ultrasounds can prompt the surfactant molecule penetrate every corner of the multiphase medium. Therefore, the surfactant molecule can easily reach the surface of the medium, and the oil production rate is improved.

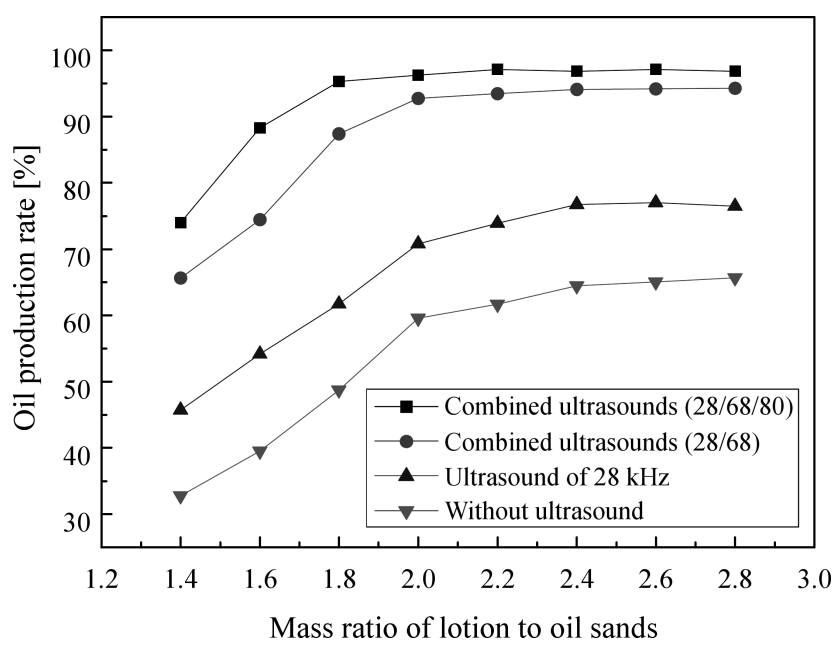

Fig. 6. Effect of mass ratio of lotion to oil sands on oil production rate.

\subsubsection{Effect of temperature on oil production rate}

The temperature is also one of the important factors that affects the oil sands separation. The experimental conditions for temperature optimization are that the concentration of surfactant in the lotion is $5 \mathrm{~g} / \mathrm{l}$, the mass ratio of the lotion to oil sands is 2.4 , and the treating time is $45 \mathrm{~min}$. The relationship of the oil production rate to temperature under the four treating methods is shown in Fig. 7. From the curves, for the samples treated by dual-frequency ultrasounds, single frequency ultrasound, and mechanical stirring, it can be drawn that the oil production rate increases with the increase in treating temperature. This is because the viscosity of the asphalt on the surface of the oil sands decreases with the increase in temperature, resulting in the improvement in the flow capacity of the asphalt droplets; therefore, the asphalt and the solid particles are more favourable to be separated. However, when comes to tri-frequency ultrasounds, the oil

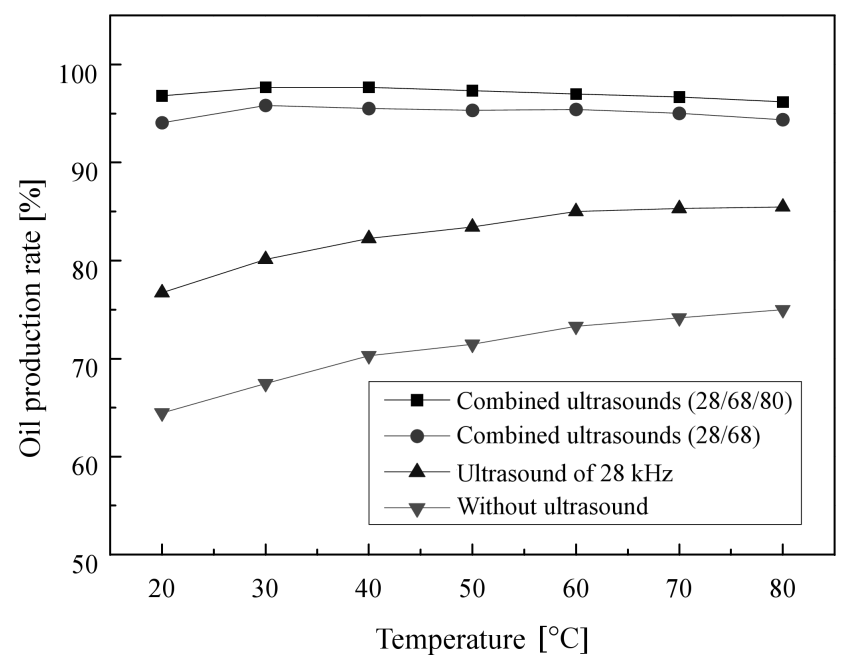

Fig. 7. Effect of temperature on oil production rate.

production rates of the tests conducted under the temperature of $20^{\circ} \mathrm{C}$ and $30^{\circ} \mathrm{C}$, are respectively $96.82 \%$ and $97.68 \%$, indicating that the maximum oil production rate can be reached even at room temperature, or the temperature has little effect on oil production rate if the sample is treated by multiple frequency ultrasounds.

\section{Conclusions}

With the introduction of multiple frequency ultrasounds into oil sands separation technology, due to the synergistic effect of the ultrasonic cavitation effect, the oil production rate of the samples treated by multiple frequency ultrasounds is higher than that of the samples treated by single frequency ultrasound. And the bigger the number of the ultrasound participating in the combination is, the higher the oil production rate of the oil sands becomes. In addition, the lower the frequency of the ultrasounds employed in the combined system is, the higher the oil production rate becomes.

Multiple frequency ultrasounds assisted lotion oil sands separation technology is promising in oil sands industry, especially as of unconventional oil and gas becoming increasingly important. The optimum treating conditions for tri-frequency ultrasounds assisted lotion oil sands separation technology are as follow: the treating time is $10-15 \mathrm{~min}$, the treating temperature is $20-30^{\circ} \mathrm{C}$, the concentration of surfactant in the lotion is $1.5 \mathrm{~g} / \mathrm{l}$, and the mass ratio of the lotion to oil sands is 1.8 .

\section{Acknowledgment}

This research is financially supported by the Fundamental Research Funds for the Central Universities (Grant 24720156035A, 24720156031A, and 16CX02018A), the National Natural Science Founda- 
tion of China (Grant 51574266 and 51474234), the Shandong Provincial Natural Science Foundation, China (ZR2014EZ002, and ZR2015EQ013).

\section{References}

1. Abramov O.V., Myasnikov S.K. (2009), Extraction of bitumen, crude oil and its products from tar sand and contaminated sandy soil under effect of ultrasound, Ultrasonics Sonochemistry, 16, 408-416.

2. Alturki A., Gates D., Maini B. (2010), On $S A G D$ in oil sands reservoirs with no cap rock, SPE Canadian Unconventional Resources and International Petroleum Conference.

3. CAO H. (2011), The study of ultrasound and surfactant technology on oily sludge elution, Engineering Science and Technology, 1, 1, 6-7.

4. Cao P., Zou W., Dai C., Chang S. (2012), A review of oil sands, Xinjiang Petroleum Geology, 33, 6, 747750 .

5. Clark K.A., Pasternack D.S. (1932), Hot water separation of bitumen form Alberta bituminous sand, Industrial and Engineering Chemistry, 24, 12, 14101416.

6. Czarnecki J., Radoev B., Schramm L., SlavCHEV R. (2005), On the nature of Athabasca oil sands, Advances in Colloid and Interface Science, 114, 53-60.

7. FEng Y. (2013), Modification and separation of oil sand with ultrasonic wave and analysis of its products, International Journal of Mining Science and Technology, 23, 531-535.

8. Fu L., Zhang G., Ge J. (2017), Study on dualfrequency ultrasounds assisted surfactant extraction of oil sands, Fuel Processing Technology, 167, 1, 146-152.

9. GATES I. (2010), Solvent-aided steam-assisted gravity drainage in thin oil sand reservoirs, Journal of Petroleum Science and Engineering, 74, 138-146.

10. JiA C., Zheng M., Zhang Y. (2012), Unconventional hydrocarbon resources in China and the prospect of exploration and development, Petroleum Exploration and Development, 39, 2, 129-136.

11. Kramers J., Mossop G. (1987), Geology and development of the Athabasca oil sand deposit, Canadian Mining and Metallurgical Bulletin, 69, 92-99.

12. Mutyala S., Fairbridge C., Jocelyn J., BelanGER J., HAWKINS R. (2010), Microwave applications to oil sands and petroleum: a review, Fuel Processing Technology, 91, 127-135.
13. Pathaka V., Babadaglia T., Edmunds N. (2011), Heavy oil and bitumen recovery by hot solvent injection, Journal of Petroleum Science and Engineering, 78, 637-645.

14. RUDYK K., SPIROV P. (2014), Upgrading and extraction of bitumen from Nigerian tar sand by supercritical carbon dioxide, Applied Energy, 113, 1397-1404.

15. Sanford E.C., Sever F.A. (1979), Processibility of Athabasca tar sands using batch extraction unit: the role of $\mathrm{NaOH}$, CIM Bull., 72, 164-169.

16. TAN J. (2011), Treatment of wastewater containing p-nitroaniline and nitrobenzene by bi-frequency ultrasonic enhanced ozonation process, Environmental Protection of Chemical Industry, 31, 1, 61-65.

17. Woods J., Kung J., Kingston D. (2012), The comparison of bitumens from oil sands with different recovery profiles, Petroleum Science and Technology, 30, $2285-2293$.

18. YAO G. (2012), Current status and development prospects for processing of Venezuelan extra-heavy crude and Canadian oil sand bitumen, Sino-Global Energy, 17, 1, 3-22.

19. Yu K., Wang Z., Jin Y., Sun Z., QIU J. (2013), Progress in oil sand treatment technology, Chemical Engineering and Machinery, 40, 1, 1-4.

20. Yun T., Li Z., Pleizier G., NG S., Chung K. (2004), Separation and characterisation of problematic solids from athabasca oil sands and waste unit samples, The Canadian Journal of Chemical Engineering, 82, 677686.

21. Zhang N., Wu S. (2010), Experimental study of sludge disintegration with dual-frequency ultrasounds, Acoustics Technical, 29, 2, 176-179.

22. Zhang S., Li W., LiU J. (2014), The technological progress in utilization of Chinese oil sands, Chemistry and Adhesion, 4, 60-64.

23. Zhang Y., Cao Z., Yang F., Xu X. (2008), World development status of oil-sands separation technology, Natural Gas Industry, 28, 12, 110-113.

24. Zhao D., Sun W., Sun M. (2011), The separating of Inner Mongolian oil sand with ultrasound, Petroleum Science and Technology, 29, 24, 2530-2535.

25. Zou C., Zhao P., Ge T., Li D., Ye H., Huang G. (2016), Bitumen recovery from Buton oil sands using a surfactant under the effect of ultrasonic waves, Energy Sources, Part A: Recovery, Utilization, and Environmental Effects, 38, 2, 270-276. 